\title{
Preliminary Study on Effect of Motor Imagery Combined With Low-Frequency Pulsed Electrical Stimulation Therapy on Upper Limb Function of Young Stroke Patients
}

\author{
Yufeng Wang ${ }^{\mathrm{a}}$, Na Chen ${ }^{\mathrm{a}}$, Jun Chen ${ }^{\mathrm{a}}$, Xiang $\mathrm{Li}^{\mathrm{a}}$, Li Sun ${ }^{\mathrm{a}}$, Yi Bao ${ }^{\mathrm{a}}$ b
}

\begin{abstract}
Background: To observe and explore the clinical effect of motor imagery therapy (MIT) combined with low-frequency pulsed electrical stimulation therapy (LFPEST) on improving upper limb function in young stroke patients.
\end{abstract}

Methods: Eighty-one young stroke patients with hemiplegia were divided into MIT group, LFPEST group and combined treatment group. Fugl-Meyer assessment (FMA), functional test hemiplegic upper extremity (FTHUE) and modified Barthel Index (MBI) were used to evaluate the upper limb function and activities of daily living before and 6 weeks after treatment.

Results: After treatment, the FMA score, FTHUE grading and MBI score of the combined treatment group and the MIT group were significantly higher than those of the LFPEST group $(\mathrm{P}<0.05)$, and the curative effect of the combined treatment group was significantly higher than that of the MIT group $(\mathrm{P}<0.05)$

Conclusions: MIT combined with LFPEST has more obvious curative effect than LFPEST and MIT, which can significantly improve the upper limb function, hand function and daily living ability of young stroke patients with hemiplegia.

Keywords: Motor imagery therapy; Low-frequency pulsed electrical stimulation therapy; Hemiplegia; Stroke

\section{Introduction}

Stroke is a disease with high mortality and disability rate. With

Manuscript submitted March 16, 2021, accepted June 30, 2021

Published online July 9, 2021

aDepartment of Neurology, Taihe Hospital Affiliated to Hubei University of Medicine, Shiyan City, Hubei Province, China

${ }^{b}$ Corresponding Author: Yi Bao, Department of Neurology, Taihe Hospital Affiliated to Hubei University of Medicine, Shiyan City, Hubei Province, China. Email:karlbaoyi@163.com

doi: https://doi.org/10.14740/jnr661 the continuous improvement of diagnosis and treatment technology, the mortality rate of cerebral infarction has decreased, but the disability rate remains high [1-3]. In the process of limb dysfunction recovery after stroke, hand function recovery is slow, which directly affects the ability of daily life and quality of life of patients; and long-term disuse will lead to muscle atrophy of hands $[4,5]$. In recent years, with the aggravation of China's aging population and the change of lifestyle, the incidence of cerebral infarction is on the rise. To make matters worse, there are more and more patients with younger age $[6$, 7]. Therefore, it is particularly important to actively seek treatment means to promote the recovery of upper limb function in stroke patients.

Motor imagery therapy (MIT) is an emerging and popular treatment method. Its principle is to consciously simulate and train a hypothetical action through the brain, and activate a specific area in the brain according to the motor memory, so as to achieve the purpose of improving motor function $[8$, 9]. Studies have confirmed that motor imagery does not depend on the residual function of patients, but is closely related to active movement. With the development of rehabilitation medicine, MIT has become an important strategy for exercise function rehabilitation, and is gradually applied to the recovery of upper limb function in stroke patients [10, 11]. Low-frequency pulsed electrical stimulation therapy (LFPEST), also known as neuromuscular electrical stimulation therapy, mainly stimulates the relevant nerves and muscles by electric current to produce passive contraction and promote the recovery of damaged neuromuscular functions $[12,13]$.

In the past research, there are many reports on limb rehabilitation treatment, but the hand rehabilitation treatment always focuses on manipulation, and the clinical effect is not good. In this study, motor imagery combined with LFPEST was used to treat young stroke patients. The recovery of upper limb function was observed and a certain curative effect was achieved, which provided the basis for exploring new clinical treatment approaches.

\section{Materials and Methods}

This is a clinical prospective study. No biological specimens were used. The study was approved by the Taihe Hospital Ethics Committee, and was conducted in compliance with the eth- 
ical standards of the responsible institution on human subjects, as well as with the Helsinki Declaration.

\section{Research object and grouping}

Selection criteria included: 1) It is in accordance with the latest diagnostic criteria of acute ischemic stroke [2], ischemic stroke resulting in arm and hand weakness; 2) The age of onset was less than 50 years old; 3) Within 1 month, the disease was relatively stable with clear consciousness and no cognitive impairment; 4) There was hemiplegia of upper limbs; 5) The modified Ashworth Scale (MAS) score of upper limb elbow or wrist flexor was $\leq 1$ without taking antispasmodic drugs; 6) The revised version of kinesthetic and visual imagery questionnaire (KVIQ) score $>25 ; 7$ ) National institutes of health stroke scale (NIHSS) score $>4 ; 8$ ) Mini Mental State Examination (MMSE) score $\geq 18$; 9) Informed consent of patients was obtained.

Exclusion criteria included: 1) Severe heart, liver, kidney dysfunction and other diseases; 2) Increased intracranial pressure or acute large area cerebral infarction, bleeding tendency; 3 ) There are metal parts implanted in the body, such as cardiac pacemaker; 4) Severe aphasia; 5) Hamilton Depression Scale (HAMD) score $>7$; 6) Pain and limitation of upper extremity joints caused by various diseases, such as contracture or deformity of the upper extremity, subluxation of the shoulder.

From May 2017 to October 2019, a total of 81 stroke patients with hemiplegia who met the above criteria and were admitted to the Department of Neurological Rehabilitation, Taihe Hospital affiliated to Hubei University of Medicine were selected, all under the age of 50. According to the random number table, they were divided into MIT (27 cases), LFPEST (27 cases) and combination therapy group (27 cases).

\section{Basic treatment methods}

All patients received basic treatment: conventional drug therapy, routine rehabilitation training and traditional therapy (acupuncture, massage, etc.) twice a day for 45 min, 6 days a week for 6 weeks.

\section{Conventional drug treatment}

Conventional drug treatment included anti-platelet aggregation, lowering blood lipid and stabilizing plaques, improving blood circulation, nourishing nerve, scavenging oxygen free radicals, promoting collateral circulation establishment, etc.

\section{Routine rehabilitation training}

The therapist selected the following rehabilitation training according to the patient's functional impairment: the placement of good limbs on the bed in the early stage; passive movement and stretching therapy of affected side limbs; the non-hemiple- gic side drives the hemiplegic side mainly with grinding board, roller and inserting rod training from coarse to fine; daily living ability training (including dressing and undressing, grasping, housework, eating with chopsticks, combing hair with comb, etc.).

\section{Group treatment methods}

On the premise of basic treatment, the three groups were given LFPEST, MIT and LFPEST combined with MIT respectively.

\section{Treatment of LFPEST}

Low-frequency pulse electrical stimulation was performed with PHYSIOMED CE1275 low-frequency therapeutic apparatus (Germany). In sitting or supine position, 75\% medical alcohol was used to degrease the local skin. Circular electrodes with a diameter of $2 \mathrm{~cm}$ were placed on the abdomen of the muscles related to the upper limbs of hemiplegic patients. The positive electrodes were placed on the back of the wrist, and the negative electrodes were placed on the extensor carpi radialis longus and brevis, abductor pollicis brevis, flexor pollicis brevis, opponens pollicis, and adductor pollicis. Treatment parameters: the waveform is a double symmetrical square wave, frequency $40-80 \mathrm{~Hz}$, pulse width $200-500 \mu \mathrm{S}$, intensity was $10-30 \mathrm{~mA}$, continuous treatment for $3-15 \mathrm{~S}$, resting time for $6-75 \mathrm{~S}$, the intensity should cause wrist joint back extension, and the patient can tolerate it. Low-frequency pulse electrical stimulation was performed twice a day for 20 min for 6 weeks.

\section{Treatment of MIT}

The patient relaxed in a quiet environment, took the sitting position, and the height of the table top was suitable for the patient's comfort. The occupational therapist explained and demonstrated the whole imaginary movement process, the exercise tasks included fist holding extension, wrist joint back extension and flexion, thumb and index finger opposition movement, thumbs up, make the "OK" gesture, holding the pen, grasping the ball, moving the wooden stick, pouring water from the cup, transferring the marbles with the spoon, etc. First, let the patient complete the specified action with the healthy side upper limb, so that the patient can master the correct movement mode and repeatedly imagine the specified action process. Guide the patient to imagine the movement, and focus all attention on the affected side of the body. After completing one exercise, relax, and then the patients exercise again, repeat 10 - 20 times. During the treatment, pay attention to the patient's safety, avoid high tension and increased muscle tension; and the patients exercise twice a day, 40 min each time, 6 days a week for 6 weeks. During the treatment, the therapist can gradually increase the difficulty of the task according to the functional status of the patient. If the patient has discomfort symptoms such as pain and fatigue, which makes it impossible to carry out normal exercise training, the training must be terminated immediately. 
Table 1. Comparison of General Data of Patients in the Three Groups When Enrolled

\begin{tabular}{llll}
\hline & MIT group & LFPEST group & Combination therapy group \\
\hline Number of cases (n) & 27 & 27 & 27 \\
Gender (n) & 15 & 14 & 15 \\
$\quad$ Male & 12 & 13 & 12 \\
$\quad$ Female & $44.74 \pm 9.25$ & $45.48 \pm 7.93$ & $44.96 \pm 8.32$ \\
Age, mean \pm SD (years) & $4.11 \pm 2.08$ & $3.67 \pm 1.92$ & $3.55 \pm 1.89$ \\
Course, mean \pm SD (days) & & & 12 \\
Hemiplegia (n) & 11 & 13 & 15 \\
$\quad$ Left & 16 & 14 & 13 \\
\hline
\end{tabular}

There was no significant difference among the three groups. MIT: motor imagery therapy; LFPEST: low-frequency pulsed electrical stimulation therapy; SD: standard deviation.

\section{Combination therapy}

On the basis of MIT combined with LFPEST, the treatment method and duration were the same as those in the MIT group.

\section{Evaluation method and index}

The upper limb function and activities of daily living (ADL) were assessed by the same professional trained rehabilitation assessor before and 6 weeks after treatment with Fugl-Meyer assessment (FMA), Hong Kong version of functional test hemiplegic upper extremity (FTHUE) and modified Barthel Index (MBI).

\section{FMA score}

The Fugl-Meyer scale upper part (FMA-UE) was used to evaluate the motor function of the patient's upper limb, including reflex activity, flexor joint movement, extensor joint movement, activity with joint movement, separation movement, reflex activity detection, wrist stability, hand movement, hand coordination and speed detection, a total of 33 evaluation indicators. Each item is scored according to three levels ( 0 - 2 points: 0 points means that cannot be completed, 1 point means partial completion, 2 points means complete); the full score is 66 points, the higher the score, the better the motor function of the patient's upper limbs.

\section{FTHUE grading}

Hong Kong version of FTHUE classification is divided into seven levels; each level has two activities to test; each activity is completed within $3 \mathrm{~min}$; and there are three attempts. According to the requirements of each grade, the physician determined whether the patient has completed the level test. The result is that the upper limb function of the patient is at a certain level. The higher the level is, the better the upper limb function of the patient.
MBI score

The MBI rating scale includes 10 activities of daily living, with a minimum score of 0 , a maximum score of 5 - 15; and a full score of $10-20$ points were classified as extremely serious functional defects, 25 - 45 points as severe functional defects, 50 - 70 points as moderate functional defects, 75 - 95 points as mild functional defects, and 100 points as being able to take care of themselves, according to the completion of patients and self-care ability to score.

\section{Statistical methods}

SPSS 20.0 statistical software was used to analyze and process the data. The measurement data were expressed by (mean \pm standard deviation (SD)), the analysis of variance was used for intergroup comparison, paired $t$-test was used for intragroup comparison, rank sum test was used for non-normal distribution data, Chi-square test was used for counting data, and rank sum test was used for grade data. $\mathrm{P}<0.05$ means the difference is statistically significant.

\section{Results}

There was no significant difference in the number of cases, gender, age, average course of disease, MMSE score, NIHSS score, stroke type and hemiplegic side among the three groups $(\mathrm{P}>0.05$, Table 1).

\section{The result of FMA score}

Before treatment, there was no significant difference in FMA scores among the three groups $(\mathrm{F}=0.679, \mathrm{P}>0.05)$; the FMA scores of the three groups after treatment were significantly higher than those before the treatment in the group $(\mathrm{P}<0.01)$; after treatment, the FMA scores of the combined treatment group were significantly higher than those of the MIT group, 
Table 2. Comparison of FMA Scores of Right Upper Limb Function Among Three Groups Before and After Treatment

\begin{tabular}{llll}
\hline \multirow{2}{*}{ Group } & Number of cases (n) & \multicolumn{2}{c}{ FMA score (mean \pm SD) } \\
\cline { 3 - 4 } & & Before treatment & $29.85 \pm 12.41$ \\
\hline MIT & 27 & $18.29 \pm 11.57$ & $25.33 \pm 11.29$ \\
LFPEST & 27 & $17.96 \pm 10.43$ & $32.18 \pm 10.83$ \\
\hline Combined group & 27 & $17.92 \pm 9.36$ & \\
\hline
\end{tabular}

The score after treatment of combined treatment group compared with that before treatment, $\mathrm{P}<0.001$; compared with LFPEST group, $\mathrm{P}<0.001$; compared with MIT group, P < 0.001. MIT: motor imagery therapy; LFPEST: low-frequency pulsed electrical stimulation therapy; FMA: Fugl-Meyer assessment; SD: standard deviation.

and the FMA scores of the MIT group were significantly higher than those of the LFPEST group, and the differences between the groups were statistically significant $(\mathrm{P}<0.01$, Table 2).

\section{The result of FTHUE classification}

Before treatment, there was no significant difference in FTHUE grading among the three groups $(\mathrm{P}>0.05)$; after treatment, the FTHUE grading of the three groups was significantly improved, and the difference was statistically significant $(\mathrm{P}<$ 0.01); after treatment, the FTHUE grade score of the combined treatment group was better than that of the MIT group ( $\mathrm{P}<$ 0.01 ), and the MIT group was better than the LFPEST group $(\mathrm{P}<0.01$, Table 3$)$.

\section{The result of MBI score}

Before treatment, there was no significant difference in MBI scores among the three groups $(\mathrm{F}=0.383, \mathrm{P}>0.05)$; after treatment, the MBI scores of the three groups were significantly improved, and the differences were statistically significant ( $\mathrm{P}$ $<0.01$ ); after treatment, MBI scores of the combined treatment group were better than those of the MIT group $(\mathrm{P}<0.01)$, and the MBI score of the MIT group was better than that of the LFPEST group $(\mathrm{P}<0.01$, Table 4$)$.

Table 3. Comparison of FTHUE Grading of Three Groups Before and After Treatment

\begin{tabular}{|c|c|c|c|c|c|c|}
\hline \multirow[t]{2}{*}{ Grading } & \multicolumn{2}{|c|}{ MIT (n) } & \multicolumn{2}{|c|}{ LFPEST (n) } & \multicolumn{2}{|c|}{$\begin{array}{l}\text { Combined } \\
\text { group (n) }\end{array}$} \\
\hline & Before & After & Before & After & Before & After \\
\hline 1 & 8 & 0 & 10 & 0 & 11 & 0 \\
\hline 2 & 9 & 3 & 9 & 5 & 7 & 2 \\
\hline 3 & 6 & 9 & 5 & 9 & 5 & 8 \\
\hline 4 & 3 & 5 & 2 & 4 & 3 & 9 \\
\hline 5 & 1 & 6 & 1 & 5 & 1 & 6 \\
\hline 6 & 0 & 3 & 0 & 3 & 0 & 3 \\
\hline 7 & 0 & 1 & 0 & 1 & 0 & 2 \\
\hline
\end{tabular}

FTHUE: functional test hemiplegic upper extremity; MIT: motor imagery therapy; LFPEST: low-frequency pulsed electrical stimulation therapy;

\section{Discussion}

After stroke, rehabilitation exercise should be carried out as soon as possible to promote recovery, among which hand function rehabilitation exercise is the most important. However, $80 \%$ of patients have upper limb dysfunction. Hand function is the most delicate, the recovery is slow, and it is difficult to recover. If the upper limb dysfunction is still left after more than 6 months, the fine function of the upper limb will decline, and serious muscle atrophy will occur, and even the hand will be abandoned. In previous literature reports, this proportion accounts for $55-75 \%$ [14-16].

At present, there are many rehabilitation measures, including daily hand function training, acupuncture, physical therapy, pulse stimulation, MIT, etc., each of which can promote the recovery of upper limb function $[17,18]$. However, there are few reports on the efficacy of low-frequency electrical stimulation, MIT and combined therapy.

LFPEST is a treatment method that uses low-frequency pulsed current to stimulate peripheral nerve or muscle to cause muscle contraction to improve limb function. Possover used low-frequency electrical stimulation to treat 29 patients with chronic spinal cord injury. After 9 years of follow-up, 17 (68\%) of 25 patients with complete exercise-induced chronic spinal cord injury recovered upper spinal control of leg movement, making it possible to walk voluntarily [12]. In the study of Cui et al including 45 subjects, it was found that the FMA of 12-h neuromuscular electrical stimulation group was more significantly improved than that of the ordinary treatment group. This alternative therapy is easy to apply and can be used during rest or sleep in stroke patients [13]. There is now much

Table 4. Comparison of MBI Scores of Three Groups Before and After Treatment

\begin{tabular}{llll}
\hline \multirow{2}{*}{ Group } & $\begin{array}{l}\text { Number of } \\
\text { cases }(\mathbf{n})\end{array}$ & \multicolumn{2}{c}{ MBI scores $($ mean \pm SD) } \\
\cline { 3 - 4 } & 27 & $28.26 \pm 8.52$ & $49.63 \pm 10.28$ \\
\hline MIT & 27 & $27.78 \pm 7.96$ & $45.29 \pm 9.13$ \\
LFPEST & 27 & $27.59 \pm 7.23$ & $52.15 \pm 8.89$ \\
\hline Combined group & 27 & After \\
\hline
\end{tabular}

The score after treatment of combined treatment group, compared with that before treatment, $\mathrm{P}<0.001$; compared with LFPEST group, $\mathrm{P}<$ 0.001 ; compared with MIT group, $\mathrm{P}<0.001$. MBI: modified Barthel Index; MIT: motor imagery therapy; LFPEST: low-frequency pulsed electrical stimulation therapy; SD: standard deviation. 
experience with low-frequency electrical stimulation [12, 19, 20]. Therefore, in this study, LFPEST was used to rehabilitate the upper limbs of stroke patients. The results showed that the FMA, FTHUE, MBI scores of the patients in the LFPEST group were significantly improved, and the upper limb function was significantly improved.

MIT is mainly aimed at some patients with hemiplegia whose upper limbs cannot move actively. It can significantly improve the sense of active participation and cooperation of patients by consciously simulating a certain action without obvious limb activities. The more thoroughly the patients are familiar with, understand and remember the movements, the more they can activate a specific motor area by motor memory in the brain, which provides an effective treatment method for the early rehabilitation training of patients $[8,11]$. In the study of Jin hyunck Park, after intervention, it was found that both mental imagery training combined with electromyogramtriggered neuromuscular electrical stimulation and electromyogram-triggered neuromuscular electrical stimulation in 68 subjects could improve upper limb function and activities of daily living in patients with chronic stroke [21].

In the clinical practice of MIT, it is difficult to monitor the content of patient's motor imagination. Whether the therapist's motor instructions are effectively implemented by patients is the key to the success or failure of treatment. Therefore, it is very important to improve the ability of patient's motor imagination. This study is mainly aimed at young patients, and with the MMSE score $\geq 18$, as shown in the inclusion criteria, which is based on this consideration. In order to improve the compliance, this study mainly used simple actions in daily life, and repeated training and strengthening, in order to form a motor memory process in the brain and improve the curative effect. The results showed that FMA, FTHUE and MBI scores of patients in the MIT group were significantly improved, and the upper limb function was significantly improved, which was consistent with the results of Jin hyunck Park [21].

Previous studies have found that low-frequency pulsed therapy and MIT can effectively improve upper limb function of hemiplegic patients $[8,10,12]$. However, there is no clear literature report on the efficacy and treatment standard of MIT combined with LFPEST.

The results showed that the FMA, FTHUE and MBI scores of the combined treatment group and the MIT group after 6 weeks of treatment were significantly higher than those of the LFPEST group $(\mathrm{P}<0.05)$; and the FMA, FTHUE and MBI scores of the combined treatment group were significantly improved than those of the MIT group $(\mathrm{P}<0.05)$. It can significantly improve the upper limb function of stroke patients with hemiplegia, and it is more obvious than low-frequency pulsed therapy and MIT. It can improve the internal muscle atrophy of stroke patients, improve hand function and daily life selfcare ability. In our study, the efficacy of combination therapy is the best. There are two possible reasons: first, combination therapy may have synergistic effect and increase the efficacy, but the mechanism needs further study; second, the sample size is small, there may be bias, need to expand the sample size to confirm.

This study does have limitations. First, the sample size used is too small. Second, each patient's educational level and understanding are different, which may affect the effect of rehabilitation treatment. Third, according to the literature reports [22], the treatment effect of different gender is different, but this study does not distinguish the difference of treatment effect between men and women. There are two main reasons. First, the number of specimens is too small to show the difference; second, this was an open-label study and this could have also biased the results.

\section{Conclusions}

LFPEST combined with MIT is not a mechanical addition, and can be carried out at the same time. It is safe and effective, with fewer adverse reactions, and is easy to be accepted. This therapy can mobilize the subjective initiative to the greatest extent, and can effectively improve the upper limb function of stroke patients with hemiplegia. The curative effect is better than MIT and LFPEST, which is worthy of clinical application.

\section{Acknowledgments}

We thank all the participants for their enthusiastic participation in this study.

\section{Financial Disclosure}

The study is supported by the National Natural Science Foundation of China (grant number: 81671238).

\section{Conflict of Interest}

None to declare.

\section{Informed Consent}

The informed consents were obtained.

\section{Author Contributions}

YFW, NC and YB performed the data analyses, statistical analyses, and wrote the paper. YB and JC involved in the study design. JC, XL and LS oversaw data collection. NC and XL assisted with the implementation of data collection. YB provided input into the statistical analysis design. All authors read and approved the final manuscript.

\section{Data Availability}

The data supporting the findings of this study are available from the corresponding author upon reasonable request. 


\section{References}

1. Truelsen T, Krarup LH, Iversen HK, Mensah GA, Feigin VL, Sposato LA, Naghavi M. Causes of death data in the global burden of disease estimates for ischemic and hemorrhagic stroke. Neuroepidemiology. 2015;45(3):152160.

2. Ji R, Schwamm LH, Pervez MA, Singhal AB. Ischemic stroke and transient ischemic attack in young adults: risk factors, diagnostic yield, neuroimaging, and thrombolysis. JAMA Neurol. 2013;70(1):51-57.

3. Bao Y, Du X, Zhang M, et al. Progress in the treatment of acute ischemic stroke, current challenges and the establishment of clinical decision-making system. Journal of Neurology Research. 2019;9(4-5):51-59.

4. Cunha BP, de Freitas S, de Freitas PB. Assessment of the ipsilesional hand function in stroke survivors: the effect of lesion side. J Stroke Cerebrovasc Dis. 2017;26(7):16151621.

5. Lum PS, Godfrey SB, Brokaw EB, Holley RJ, Nichols D. Robotic approaches for rehabilitation of hand function after stroke. Am J Phys Med Rehabil. 2012;91(11 Suppl 3):S242-254.

6. Wang J, Bai L, Shi M, Yang L, An Z, Li B, Zhao W, et al. Trends in age of first-ever stroke following increased incidence and life expectancy in a low-income Chinese population. Stroke. 2016;47(4):929-935.

7. Guo X, Li Z, Zhou Y, Yu S, Yang H, Zheng L, Liu Y, et al. Metabolic profile for prediction of ischemic stroke in Chinese hypertensive population. J Stroke Cerebrovasc Dis. 2019;28(4):1062-1069.

8. Herrador Colmenero L, Perez Marmol JM, Marti-Garcia C, Querol Zaldivar MLA, Tapia Haro RM, Castro Sanchez AM, Aguilar-Ferrandiz ME. Effectiveness of mirror therapy, motor imagery, and virtual feedback on phantom limb pain following amputation: A systematic review. Prosthet Orthot Int. 2018;42(3):288-298.

9. Malouin F, Richards CL, Durand A, Doyon J. Clinical assessment of motor imagery after stroke. Neurorehabil Neural Repair. 2008;22(4):330-340.

10. Kho AY, Liu KP, Chung RC. Meta-analysis on the effect of mental imagery on motor recovery of the hemiplegic upper extremity function. Aust Occup Ther J. 2014;61(2):38-48.

11. Harris JE, Hebert A. Utilization of motor imagery in upper limb rehabilitation: a systematic scoping review. Clin Rehabil. 2015;29(11):1092-1107.
12. Possover M. Ten-year experience with continuous lowfrequency pelvic somatic nerves stimulation for recovery of voluntary walking in people with chronic spinal cord injury: a prospective case series of 29 consecutive patients. Arch Phys Med Rehabil. 2021;102(1):50-57.

13. Cui BJ, Wang DQ, Qiu JQ, Huang LG, Zeng FS, Zhang Q, Sun M, et al. Effects of a 12-hour neuromuscular electrical stimulation treatment program on the recovery of upper extremity function in sub-acute stroke patients: a randomized controlled pilot trial. J Phys Ther Sci. 2015;27(7):2327-2331.

14. Langhorne P, Bernhardt J, Kwakkel G. Stroke rehabilitation. Lancet. 2011;377(9778):1693-1702.

15. Dobkin BH. Strategies for stroke rehabilitation. Lancet Neurol. 2004;3(9):528-536.

16. Maceira-Elvira P, Popa T, Schmid AC, Hummel FC. Wearable technology in stroke rehabilitation: towards improved diagnosis and treatment of upper-limb motor impairment. J Neuroeng Rehabil. 2019;16(1):142.

17. Gittler M, Davis AM. Guidelines for adult stroke rehabilitation and recovery. JAMA. 2018;319(8):820-821.

18. McDonald MW, Black SE, Copland DA, Corbett D, Dijkhuizen RM, Farr TD, Jeffers MS, et al. Cognition in stroke rehabilitation and recovery research: Consensusbased core recommendations from the second Stroke Recovery and Rehabilitation Roundtable. Int J Stroke. 2019;14(8):774-782.

19. Chan KM, Curran MW, Gordon T. The use of brief post-surgical low frequency electrical stimulation to enhance nerve regeneration in clinical practice. J Physiol. 2016;594(13):3553-3559.

20. Tosun A, Ture S, Askin A, Yardimci EU, Demirdal SU, Kurt Incesu T, Tosun O, et al. Effects of low-frequency repetitive transcranial magnetic stimulation and neuromuscular electrical stimulation on upper extremity motor recovery in the early period after stroke: a preliminary study. Top Stroke Rehabil. 2017;24(5):361-367.

21. Park JH. Effects of mental imagery training combined electromyogram-triggered neuromuscular electrical stimulation on upper limb function and activities of daily living in patients with chronic stroke: a randomized controlled trial. Disabil Rehabil. 2020;42(20):2876-2881.

22. Arboix A, Cartanya A, Lowak M, Garcia-Eroles L, Parra $\mathrm{O}$, Oliveres M, Massons J. Gender differences and woman-specific trends in acute stroke: results from a hospital-based registry (1986-2009). Clin Neurol Neurosurg. 2014;127:19-24. 